\section{Considering the risks and safety of intraperitoneal injections}

\section{To the Editor:}

The November 2016 issue of Lab Animal included an article by Baek and colleagues ${ }^{1}$ describing a novel technique for handling mice while administering intraperitoneal injections. I commend the authors for their efforts to develop new methods for these injections in mice. The authors report that their new method involves moderately less stress, as measured by adrenocorticotropic hormone and corticosterone assays. Test values for corticosterone levels generally rise when handling mice, and corticosterone levels are a common surrogate indicator of stress. However, the relative costs of stress and harm, or risk of harm, must be considered in the context of each method. It is difficult to justify the introduction of a new method without providing detailed evidence of its safety.

In my opinion, a major concern is that the proposed new method introduces a partially blind element into the process of administering an intraperitoneal injection. In this proposed method, the mouse is allowed to grab a cage surface with its front legs; the investigator holds the right hind limb to stretch the mouse straight and then administers the injection, as Baek and colleagues describe ${ }^{1}$. This introduces several uncontrolled variables. First, it appears that the investigator might not have a clear view of the injection site. Second, mice of different strains and sexes will vary with respect to their capacity to grasp a cage with their front legs. Third, because this method involves minimal physical restraint, the investigator's comfort and experience with handling and injecting mice becomes a more influential variable. Finally, because this method relies on the strength of the mouse's grasp, it appears that the investigator has a limited amount of time within which to safely administer the injection before the mouse releases its grip or changes its posture.

These uncontrolled variables are of concern because a misplaced intraperitoneal injection can puncture a rodent's cecum, which can lead to peritonitis and morbidity ${ }^{2}$. I have an impression, based on my clinical experience administering intraperitoneal injections of anesthesia in mice and rats, that morbidity associated with intraperitoneal injections occurs with an incidence of about $1 \%$. Coria-Avila and colleagues have described the location of the cecum and the risk of cecal puncture during intraperitoneal injections, noting a similar incidence of lesions to the cecum (1.58\%) after intraperitoneal injections were administered to the lower-right quadrant of the rat's abdomen ${ }^{2}$. Their study is a valuable reminder that care is needed to prevent cecal puncture when administering injections to rodents. This study was not cited in the article by Baek and colleagues, and it remains unclear whether the proposed new method decreases the risk of cecal puncture in mice.

\section{Michael Guarnieri, PhD}

Department of Neurological Surgery, Johns Hopkins School of Medicine, Baltimore, MD. email:mguarnieri@comcast.net

1. Baek, J.M. et al. Evaluation of a novel technique for intraperitoneal injections in mice. Lab Anim. (NY) 44, 440-444 (2015).

2. Coria-Avila, G.A., Gavrila, A.M., Ménard, S., Ismail, N. \& Pfaus, J.G. Cecum location in rats and the implications for intraperitoneal injections. Lab Anim. (NY) 36, 25-30 (2007). 